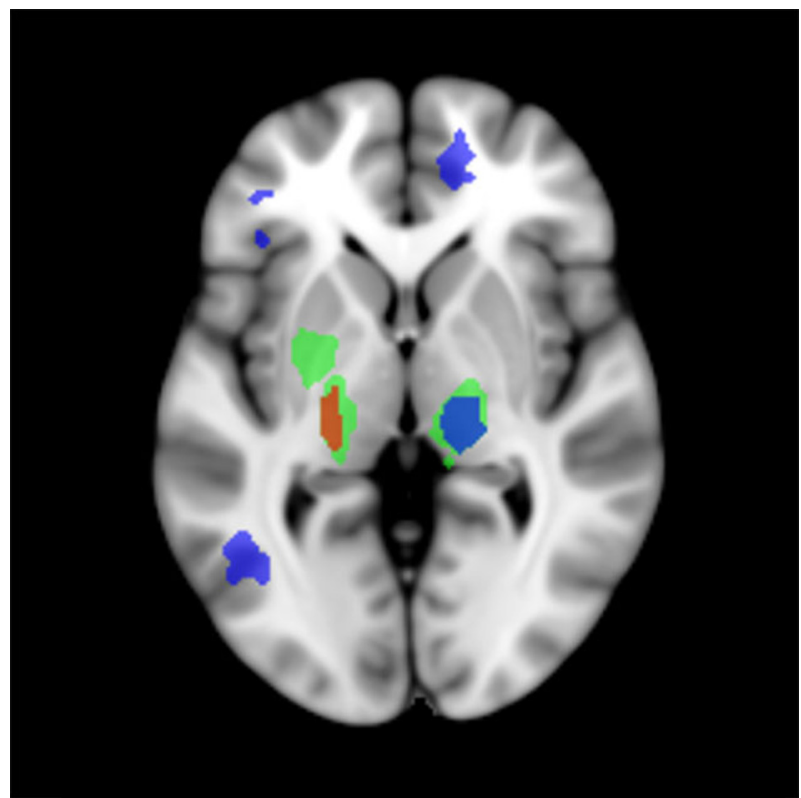

Figure 2. regions showing GM atrophyi n NC-p (in green), NC-s (in blue), and group-interaction (in red).

aminations on the last scans identified similar, but wider regions around bilateral insula with lower volume (corrected $\mathrm{p}<0.05$ ). Further LME analyses suggested that while NC-s showed more extensive atrophy during follow-up, mainly involving prefrontal and tempo-parietal cortices, atrophy of NC-p was limited to bilateral thalamus (corrected $\mathrm{p}<0.05$ ); more importantly, besides overlapping of atrophy in right thalamus, accelerated GM loss was found in clusters involving left thalamus in NC-p (uncorrected $p<0.005$, Cluster Size $>200$ ). Conclusions: The significant smaller insula, as well as accelerated thalamus atrophy, in elders who progressed MCI later, implied that subcortical limbic structures could be potential biomarkers for detecting onset of cognitive impairment.

\section{IC-P-134 INTERPLAY BETWEEN BASELINE AND \\ LONGITUDINAL CHANGE IN NEUROIMAGING MARKERS OF AMYLOID DEPOSITION AND \\ NEURODEGENERATION ACROSS THE ALZHEIMER'S DISEASE SPECTRUM}

Alexandre Bejanin, Eider M. Arenaza-Urquijo, Robin de Flores, Clémence Tomadesso, Siya Sherif, Brigitte Landeau, Gael Chetelat, Inserm,

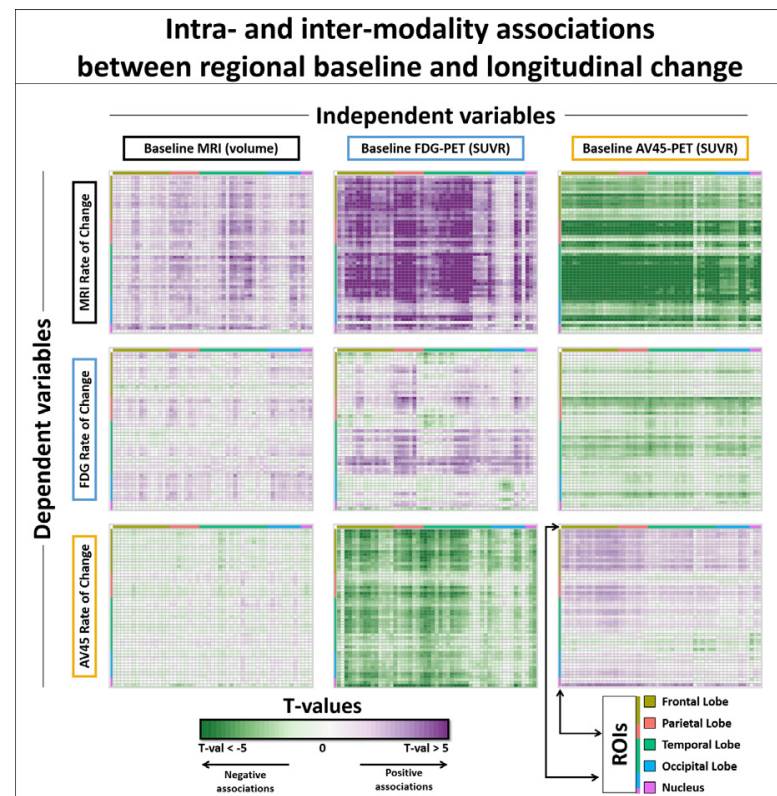

Figure 1. Heatmaps showing the significance (t-values) of intra- and intermodality associations between baseline and longitudinal change of each imaging modality. Linear mixed effects analyses were performed using $\mathrm{R}$ (nlme package) to estimate the effect of baseline on rate of change over time, whilst incorporating the longitudinal nature of the data by including within-person variation as random effects. Age at baseline and sex were included as covariates in all models. Models can be summarized as follow: - Intra-modality relationship $\rightarrow$ (Modalityl - Annual Rate of Change) $)_{\text {Region i }}$ $\sim(\text { Baseline Modalityl })_{\text {Region } \mathrm{j}}+$ Age + Sex $+(1 \mid$ Subject $)$

- Inter-modality relationship $\rightarrow$ (Modalityl - Annual Rate of Change $)_{\text {Region i }}$ $\sim(\text { Baseline Modality2 })_{\text {Region js }}+$ Age + Sex $+(1 \mid$ Subject $)$ where Modality corresponds to volume, FDG- or AV45-SUVR, and Region i and Region $j$ represent one bilateral ROIs of the Harvard-Oxford structural atlas. Note that on the heatmaps, ROIs are shown in the same order for $\mathrm{x}$ - and $y$-axis. Therefore, the upper diagonal represents the relationships when the same regions was considered (i.e. Region $i=$ Region $j$ ).

\section{Université de Caen-Normandie, Caen, France. Contacte-mail: bejanin@ cyceron. $f r$}

Background: A better understanding of baseline neuroimaging predictors of longitudinal change in amyloid deposition and neurodegeneration across the $\mathrm{AD}$ spectrum would be particularly relevant for clinical trials, disease monitoring and physiopathological mechanisms understanding. Therefore, we assessed regional

Table 1

Participant. 43 normal older adults [OA], 20 patients with subjective cognitive decline [SCD], 37 with mild cognitive impairment [MCI] and 18 with AD dementia.

\begin{tabular}{|c|c|c|c|c|c|c|c|}
\hline & \multicolumn{2}{|l|}{ OA } & \multicolumn{2}{|l|}{ SCD } & \multicolumn{2}{|l|}{ MCI } & $\mathrm{AD}$ \\
\hline $\mathrm{N}$ & 32 & 11 & 15 & 5 & 15 & 22 & 18 \\
\hline Gender (m/f) & $18 / 14$ & $4 / 7$ & $7 / 8$ & $3 / 2$ & $8 / 7$ & $8 / 14$ & $8 / 10$ \\
\hline Education (years) & $12.6 \pm 4.1$ & $10.5 \pm 3.7$ & $14 \pm 2.3$ & $13 \pm 4.9$ & $10.8 \pm 2.6$ & $11.5 \pm 4.7$ & $11.9 \pm 3.5$ \\
\hline ApoE $\varepsilon 4(\%)$ & 18.8 & 36.4 & 0 & 20 & 20 & 59.1 & 61.1 \\
\hline $\operatorname{MMSE}(/ \mathrm{BO})$ & $28.9 \pm 1.1$ & $29 \pm 0.6$ & $29.4 \pm 0.7$ & $29 \pm 1$ & $27.3 \pm 2.1$ & $26.4 \pm 1.8$ & $20.8 \pm 5.1$ \\
\hline Mattis (/144) & $141.9 \pm 2$ & $140.6 \pm 4$ & $141.7 \pm 2.5$ & $141.6 \pm 1.1$ & $135.1 \pm 5.7$ & $133 \pm 5.3$ & $119 \pm 13.6$ \\
\hline Number of visits $(1 / 2 / 3)$ & $32 / 32 / 5$ & $11 / 11 / 3$ & $15 / 15 / 4$ & $5 / 5 / 2$ & $15 / 15 / 4$ & $22 / 22 / 5$ & $18 / 18 / 0$ \\
\hline Interval visit 1-2 (months) & $19 \pm 1.3$ & $17.6 \pm 0.4$ & $22.5 \pm 2.4$ & $19.3 \pm 2.9$ & $21 \pm 0.7$ & $19.5 \pm 1.9$ & $18.5 \pm 0.6$ \\
\hline Interval visit 1-3 (months) & $37.9 \pm 5.2$ & $36.9 \pm 0.6$ & $38.4 \pm 4.4$ & $41 \pm 3.4$ & $37.4 \pm 3.5$ & $38.6 \pm 1.3$ & $n a$ \\
\hline
\end{tabular}




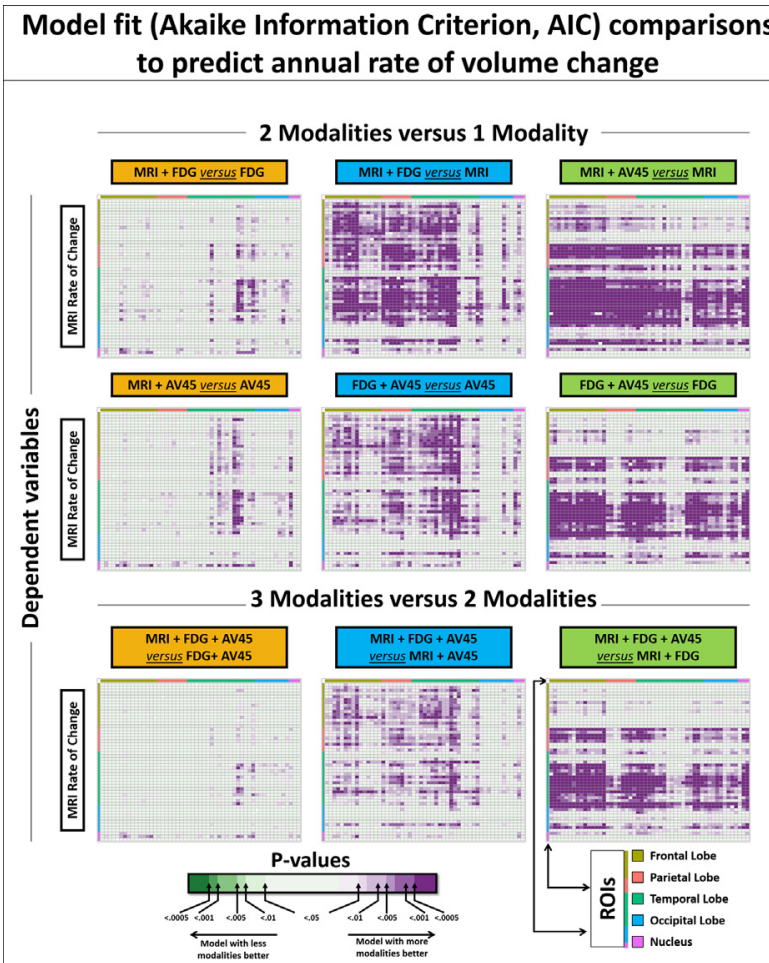

Figure 2. Heatmaps showing the significance (p-values) of the comparisons between the fit of distinct nested linear mixed effects models using the Akaike Information Criterion (AIC). Columns show the influence of including baseline volume (heatmaps of the first column), baseline FDGSUVR (heatmaps of the second column) and baseline AV45-SUVR (heatmaps of the third column) in the statistical models to predict annual rate of volume change. Linear mixed effects analyses were performed using $\mathrm{R}$ (nlme package) and included subjects as random effect, and both age at baseline and sex as covariates. Models can be summarized as follow:

-1 Modality as predictor $\rightarrow$ (Annual Rate of Volume Change $)_{\text {Region i }} \sim$ $(\text { Baseline Modalityl })_{\text {Region } j}+$ Age + Sex $+(1 \mid$ Subject $)$

-2 Modalities as predictor $\rightarrow$ (Annual Rate of Volume Change $)_{\text {Region i }} \sim$ $(\text { Baseline Modalityl })_{\text {Region j }}+(\text { Baseline Modality2 })_{\text {Regjon } j}+$ Age + Sex + (1 | Subject)

-3 Modalities as predictor (Annual Rate of Volume Change) Region i, $(\text { Baseline Modalityl })_{\text {Region } j}+(\text { Baseline Modality2 })_{\text {Regjon i }}+($ Baseline Modality3) Region j + Age + Sex $+(1 \mid$ Subject $)$

where Modality corresponds to volume, FDG- or AV45-SUVR, and Region $i$ and Region $j$ represent one bilateral ROIs of the Harvard-Oxford structural atlas. Note that on the heatmaps, ROIs are shown in the same order for $\mathrm{x}$ - and y-axis (upper diagonal: Region $i=$ Region $j$ ).

associations between baseline and longitudinal changes in volume, glucose metabolism and amyloid deposition in cognitively unimpaired and impaired older adults. Methods: 118 participants (43 normal older adults, 20 patients with SCD, 37 with MCI and 18 with $\mathrm{AD}$ dementia) underwent 2-to-3 MRI, ${ }^{18} \mathrm{~F}$-FDG-PET and ${ }^{18}$ F-AV45-PET scans (Table_1). Baseline and follow-up PET data were spatially normalized using MRI data and scaled using the brain stem and cerebral white matter respectively. The mean gray matter volume, FDG- and AV45-SUVR of 53 bilateral ROIs from the Harvard-Oxford atlas were used to perform mixed-effect models to assess intra- and inter-modality relationships between baseline and annual rate of change. Models predicting longitudinal changes with baseline values of more than one modality were also performed, and their fit was compared using the Akaike Informa- tion Criterion. Results: Intra-modality relationships revealed relatively weak associations between baseline and rate of change: the strongest relationships involved baseline medial temporal lobe volume and baseline parietal lobe metabolism (Fig_1). Inter-modality relationships showed i) weak associations with baseline volume, ii) strong relationships in fronto-parieto-temporal regions between baseline FDG-SUVR and rate of volume and AV45-SUVR changes, and iii) strong associations between baseline AV45SUVR in most brain regions and rate of volume change in frontoparieto-temporal regions. Comparisons between the fit of distinct nested models revealed that baseline volume poorly contributed to the prediction of rate of volume change when baseline FDGand/or AV45-SUVR were included in the models (Fig_2). By contrast, both baseline FDG- (especially in temporal areas) and AV45-SUVR (in most brain regions) improved the prediction of rate of volume change in tempo-parietal regions. Conclusions: Longitudinal changes in neuroimaging markers were associated in a region-specific manner with baseline metabolism versus in a non region-specific manner with baseline AV45-SUVR. Interestingly, baseline metabolism and amyloid deposition better predicted longitudinal volume changes than baseline volume, which may have clinical implications for detecting patients with the highest rate of atrophy over a follow-up period.

\section{IC-P-135 HIGH-NORMAL FASTING PLASMA GLUCOSE IN ADOLESCENCE IS ASSOCIATED WITH POORER BRAIN STRUCTURE AND FUNCTION IN MIDLIFE: THE BOGALUSA HEART STUDY}

Owen T. Carmichael ${ }^{1}$, Patrick Stuchlik ${ }^{2}$, Sreekrishna R. Pillai ${ }^{1}$, Ram Dhullipudi ${ }^{1}$, Anna Madden ${ }^{1}$, Shane Martin ${ }^{1}$, Daniel Hsia ${ }^{1}$, Vivian A. Fonseca ${ }^{2}$, Lydia Bazzano ${ }^{2},{ }^{1}$ Pennington Biomedical Research Center, Baton Rouge, LA, USA; ${ }^{2}$ Tulane University, New Orleans, LA, USA. Contact e-mail: Owen.Carmichael@pbrc.edu

Background: Fasting plasma glucose (FPG) in impaired (100-125 $\mathrm{mg} / \mathrm{dL}$ ) or diabetic $(\geq 126 \mathrm{mg} / \mathrm{dl})$ range is associated with elevated risk of damage to several end organs including the brain. However, it is not clear whether FPG at the high end of normal range confers enhanced risk of adverse brain outcomes, especially among adolescents and young adults. Methods: Fifty participants in the Bogalusa Heart Study received comprehensive evaluations of cardiometabolic risk factors periodically from childhood to midlife, followed by midlife structural and functional brain MRI and cognitive testing (age at MRI: $51.3+/-4.4$ years, see Table 1). Mean FPG per participant was calculated during the pre-20, 20-40, and over-40 periods (Figure 1). Brain MRI variables were compared between those with mean FPG during each period vs. below the pre-20 sample median $(83.5 \mathrm{mg} / \mathrm{dl})$ in models controlled for race, age, and sex. Results: The mean age at MRI was $51.3+/-4.4$ years. The sample was predominantly female (74\%) and Caucasian (74\%) (Table 1). Mean FPG was in the impaired range for 0,2 , and 9 individuals in the pre-20, 20-40, and over 40 periods (Table 2). Pre-20 mean FPG above median value was associated with significantly greater MRI-based white matter hyperintensity $(\mathrm{WMH})$ volume $(\mathrm{p}=.015$, Figure 2) and significantly less fMRI activation to a Stroop task ( $\mathrm{p}=.043$, Figure 3 ) on the midlife MRI, compared to mean FPG below median value. Mean FPG above median in the $20-40$ period was associated with significantly greater WMH volume $(\mathrm{p}=.02)$ and less gray matter volume $(\mathrm{p}=.043)$, with a trend toward less Stroop activation $(\mathrm{p}=.085)$ on the midlife MRI, compared to 